\title{
Primeira ocorrência de Tupinambis quadrilineatus Manzani \& Abe, 1997 (Squamata: Teiidae) no bioma Amazônia
}

First occurrence of Tupinambis quadrilineatus Manzani \& Abe, 1997 (Squamata: Teiidae) in the Amazonian Biome

\author{
Leandro Valle Ferreiral \\ Jorge Luiz Gavina Pereirall \\ Teresa Cristina Sauer Avila-Pires ${ }^{\text {III }}$ \\ Priscilla Prestes Chaves ${ }^{\mathrm{IV}}$ \\ Denise de Andrade Cunhav \\ Camila da Silva Furtado vi
}

Resumo: É relatada a ocorrência de Tupinambis quadrilineatus Manzani \& Abe, 1997 (Squamata: Teiidae) no bioma Amazônia, em vegetação de cerrado no sudeste do Pará, Brasil. Nesta região, estão localizados fragmentos de cerrado de grandes dimensões e situados fora do atual sistema de áreas protegidas do estado. T. quadrilineatus havia sido registrada somente nos estados de Goiás, Mato Grosso, Tocantins e Minas Gerais, no bioma Cerrado. Esse é o primeiro registro da espécie nos cerrados do bioma Amazônia e no estado do Pará. Esta descoberta reforça a ideia de que o conhecimento sobre a distribuição de espécies na Amazônia é ainda incompleto. Recomenda-se que novos inventários sejam priorizados para esta região e que partes dos fragmentos de cerrado situados no sudeste do estado sejam usadas para a criação de novas unidades de conservação.

Palavras-chave: Amazônia. Lagartos. Cerrado. Conservação.

Abstract: We here report the occurrence of Tupinambis quadrilineatus Manzani \& Abe, 1997 (Squamata: Teiidae) in the Amazonian biome, in vegetation of savanna situated in southeastern Pará, Brazil. This region has extensive fragments of savanna situated outside any protected area of Pará. T. quadrilineatus was only known from Mato Grosso, Goiás, Tocantins and Minas Gerais states, within the Cerrado biome. This is the first report of the species in savanna in the Amazonian biome and in the state of Pará. This discovery reinforces the idea that knowledge about Amazonian biodiversity is still insufficient. We recommend that new inventories be made in the region and that savanna fragments in southeastern Pará be incorporated into new protected areas.

Keywords: Amazon. Lizards. Savanna. Conservation.

l Museu Paraense Emílio Goeldi. Coordenação de Ciências da Terra e Ecologia. Belém, Pará, Brasil (lvferreira@museu-goeldi.br).

" Museu Paraense Emílio Goeldi. Coordenação de Pesquisa e Pós-Graduação. Belém, Pará, Brasil (jorgegavina@museu-goeldi.br).

III Museu Paraense Emílio Goeldi. Coordenação de Zoologia. Belém, Pará, Brasil (avilapires@museu-goeldi.br).

v Museu Paraense Emílio Goeldi. Belém, Pará, Brasil (priscillapch2004@yahoo.com.br).

$\checkmark$ Universidade Federal Rural da Amazônia. Belém, Pará, Brasil (deni_bio@yahoo.com.br).

vı Museu Paraense Emílio Goeldi. Belém, Pará, Brasil (kmila_furtado@̄yahoo.com.br).

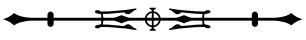




\section{INTRODUÇÃO}

O conhecimento sobre a distribuição de espécies no Brasil e principalmente na Amazônia é ainda limitado e desigual, tanto em relação à classificação biológica quanto em termos de distribuição geográfica (Lewinsohn \& Prado, 2002; Silva et al., 2001), ainda que venha progressivamente aumentando. No contexto amazônico, pode-se dizer que lagartos são relativamente bem conhecidos na Amazônia brasileira (Avila-Pires, 1995), ainda que novas espécies e novos registros ocorram com frequência. Relatamos, aqui, o primeiro registro no bioma Amazônia do lagarto Tupinambis quadrilineatus Manzani \& Abe, 1997, encontrado em fragmentos de vegetação de cerrado no sudeste do Pará, em 29 de abril de 2009 , no período da manhã. $\bigcirc$ animal estava em repouso, escondido na camada herbácea da vegetação de cerrado, o dia estava nublado e o céu apresentava poucas nuvens.

Essa espécie de lagarto foi descrita em 1997, como endêmica do bioma Cerrado, nos estados de Goiás, Mato Grosso e Tocantins (Manzani \& Abe, 1997). Em 1998, Colli et al. (1998) descreveram a espécie Tupinambis cerradensis, procedente dos estados de Mato Grosso e Goiás, um sinônimo de T. quadrilineatus, conforme os próprios autores reconheceram em uma nota adicionada durante as provas do trabalho. A distribuição atual conhecida da espécie foi revisada por Silveira (2009), incluindo, além dos estados já mencionados, Minas Gerais. $\bigcirc$ ponto mais ao norte indicado é a região de Jalapão (10`32' 46,69" S; 46²5' 13,20" W), no estado de Tocantins, com base em registros de Vitt et al. (2005) e Mesquita et al. (2006). A descoberta desta espécie nos cerrados do sudeste do Pará é o primeiro registro da espécie para o bioma Amazônia, reforçando a importância de inventários biológicos nesta região que, atualmente, apresenta alto grau de degradação ambiental.

\section{MATERIAL E MÉTODOS}

O espécime registrado neste estudo foi localizado em um fragmento de vegetação de cerrado. Esta vegetação é denominada de Savana Estacional Arborizada (Veloso et al., 1991), com cerca de 180 mil hectares entre os municípios de Redenção e Santa Maria das Barreiras (Figura 1). $\bigcirc$ exemplar (adulto) não foi coletado, sendo a identificação taxonômica feita com base no registro fotográfico (Figura 3).

\section{RESULTADOS E DISCUSSÃO}

A vegetação de cerrado ocupa somente 2,3\% da área do Pará, ocorrendo em manchas isoladas no norte do estado, ilha do Marajó, margens dos rios Amazonas, Tapajós e Tocantins, no sudoeste e sudeste do estado (Figura 1).

Esse tipo de vegetação é caracterizado pela presença de uma espessa camada de gramíneas e plantas herbáceas de diversas espécies e árvores tortuosas, bastante espaçadas, não formando um dossel contínuo (Figura 2).

O exemplar de T. quadrilineatus foi registrado neste ambiente, onde se encontrava em repouso (Figura 3).

Silveira (2009) registrou esta espécie em 18 municípios do bioma Cerrado, nos estados do Distrito Federal (um registro), Goiás (seis registros), Minas Gerais (dois registros), Mato Grosso (quatro registros) e Tocantins (dois registros). $O$ encontro desta espécie no bioma Amazônia, no estado do Pará, amplia a distribuição da espécie em mais de 300 km em direção norte (8॰ 04' 33" S; 49॰ 58' 42" W) (Figura 4).

A descoberta de T. quadrilineatus na Amazônia reforça a importância de serem ampliados os inventários biológicos, a fim de aumentar o conhecimento sobre a distribuição de espécies na região, principalmente em tipos de vegetação ameaçados pela expansão humana, tais como os cerrados do estado do Pará, ameaçados pela agricultura de grãos e pela pecuária extensiva.

Os fragmentos de cerrado visitados neste estudo estão localizados em regiões classificadas pelo Macrozoneamento Ecológico-Econômico do Pará como "zonas de consolidação", conforme citado na Lei do

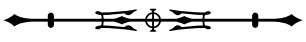




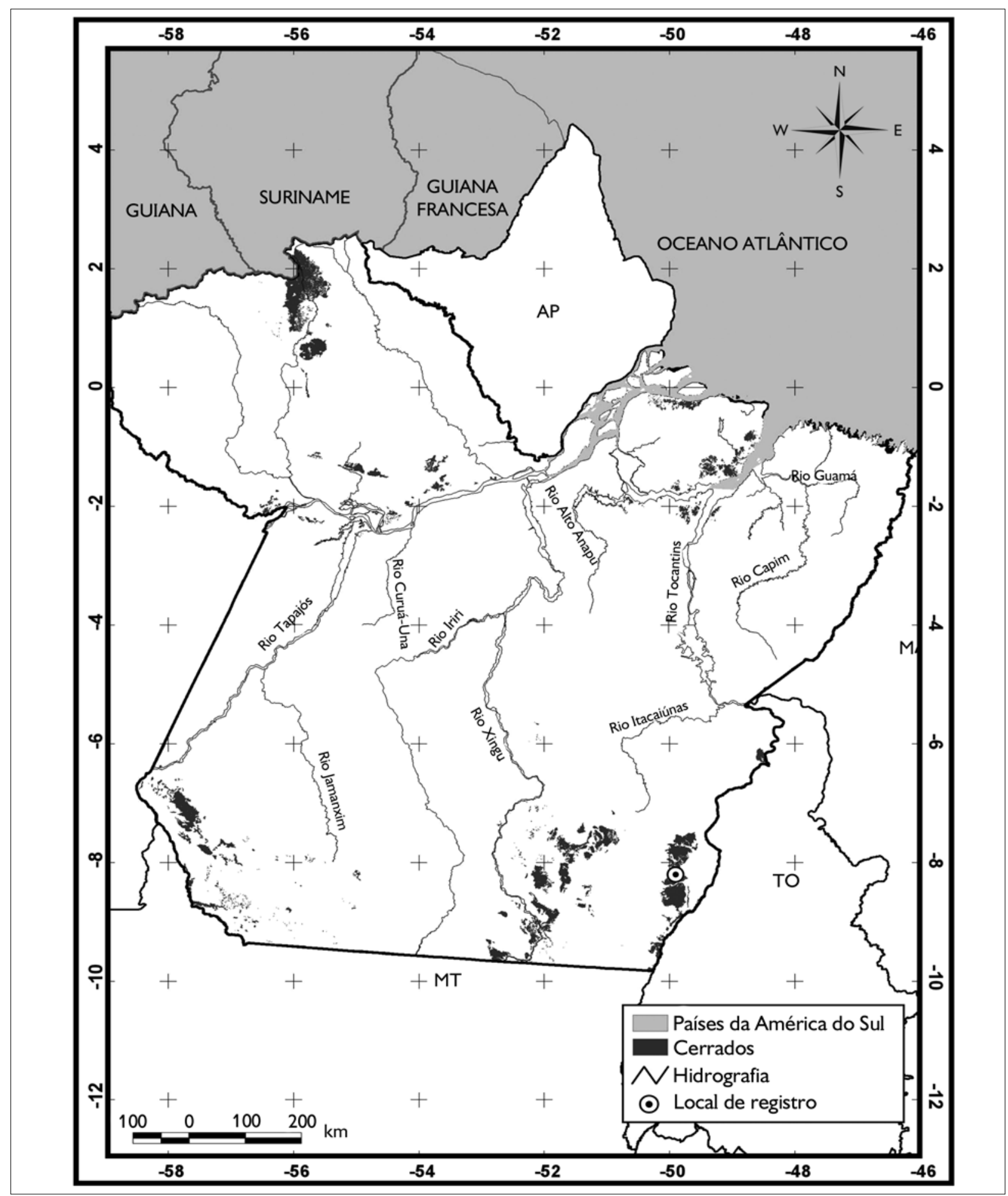

Figura 1. Distribuição das savanas (cerrado) no estado do Pará, mostrando a área onde foi registrada a espécie Tupinambis quadrilineatus Manzani \& Abe, 1997. Fonte: Leandro Ferreira (MPEG).

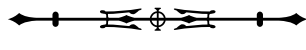




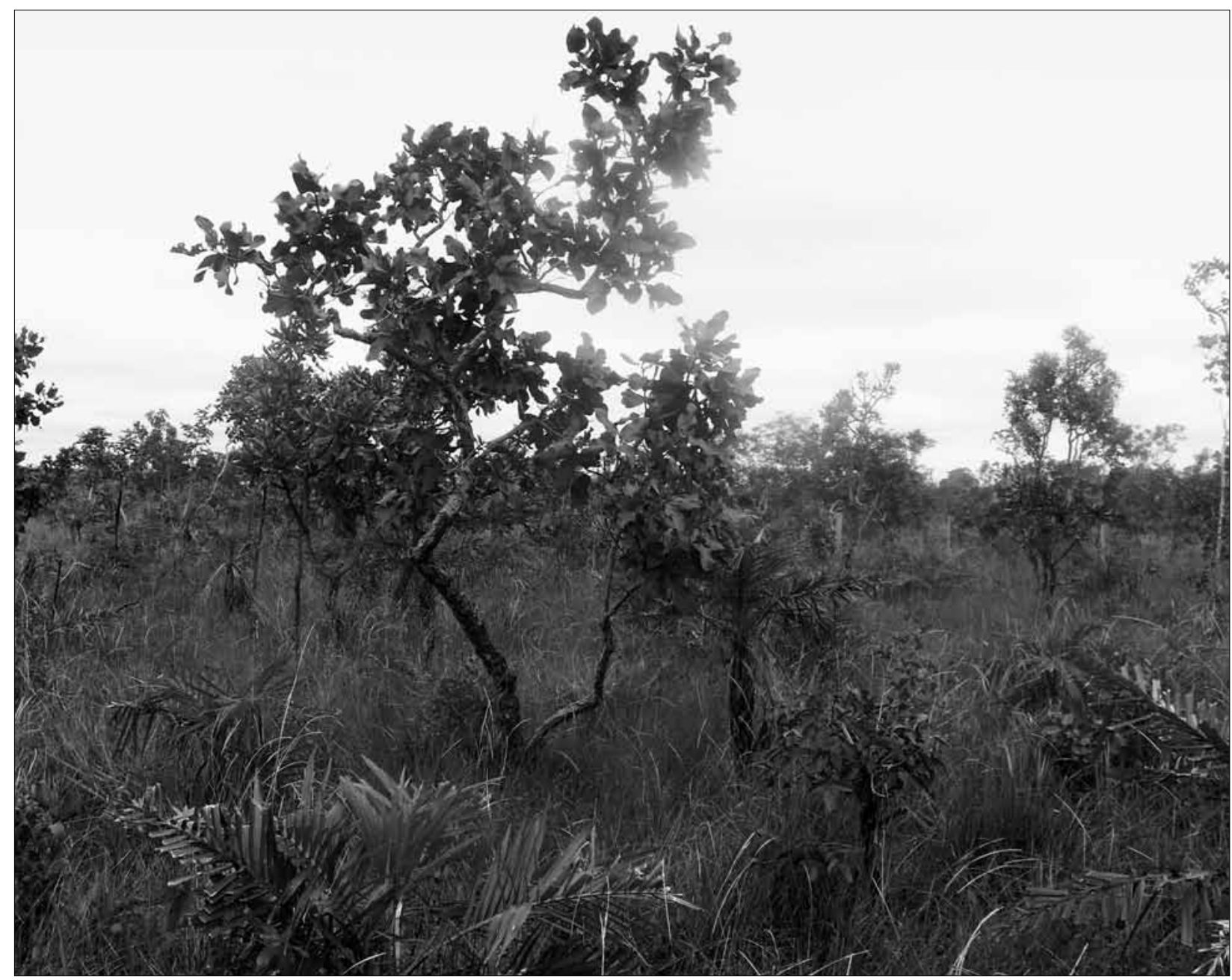

Figura 2. Vegetação de cerrado, classificada como Savana Estacional Arborizada (Veloso et al., 1991), local onde foi encontrado exemplar de Tupinambis quadrilineatus Manzani \& Abe, 1997. Foto: Leandro Ferreira (MPEG).

Macrozoneamento $-\mathrm{n}^{\circ} \cdot 6.745$, de 6 de maio de 2005 (SEMA, 2009). De acordo com o artigo $5^{\circ}$ da Lei do Macrozoneamento, a zona destinada à consolidação das atividades produtivas deverá incluir as áreas alteradas ou que apresentam degradação da qualidade ambiental e será objeto de zoneamento ecológico-econômico em escala de detalhe, a ser realizado de acordo com prioridades definidas pelo Poder Executivo, observada a legislação aplicável (ZEE, 2004). A fim de atender o artigo $5^{\circ}$ da Lei do Macrozoneamento, o Museu Paraense Emílio Goeldi (MPEG), em parceria com a Universidade Federal do Pará, está realizando estudos sobre a biodiversidade na zona de consolidação. Entre esses estudos, os inventários biológicos são parte fundamental para aumentar o conhecimento sobre a distribuição da fauna e da flora no estado, principalmente nos encraves de vegetação aberta, regiões pouco inventariadas.

\section{CONCLUSÃO}

A descoberta da espécie Tupinambis quadrilineatus Manzani \& Abe, 1997 (Squamata: Teiidae) em fragmentos de vegetação de cerrado no sudeste do

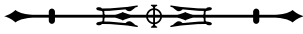




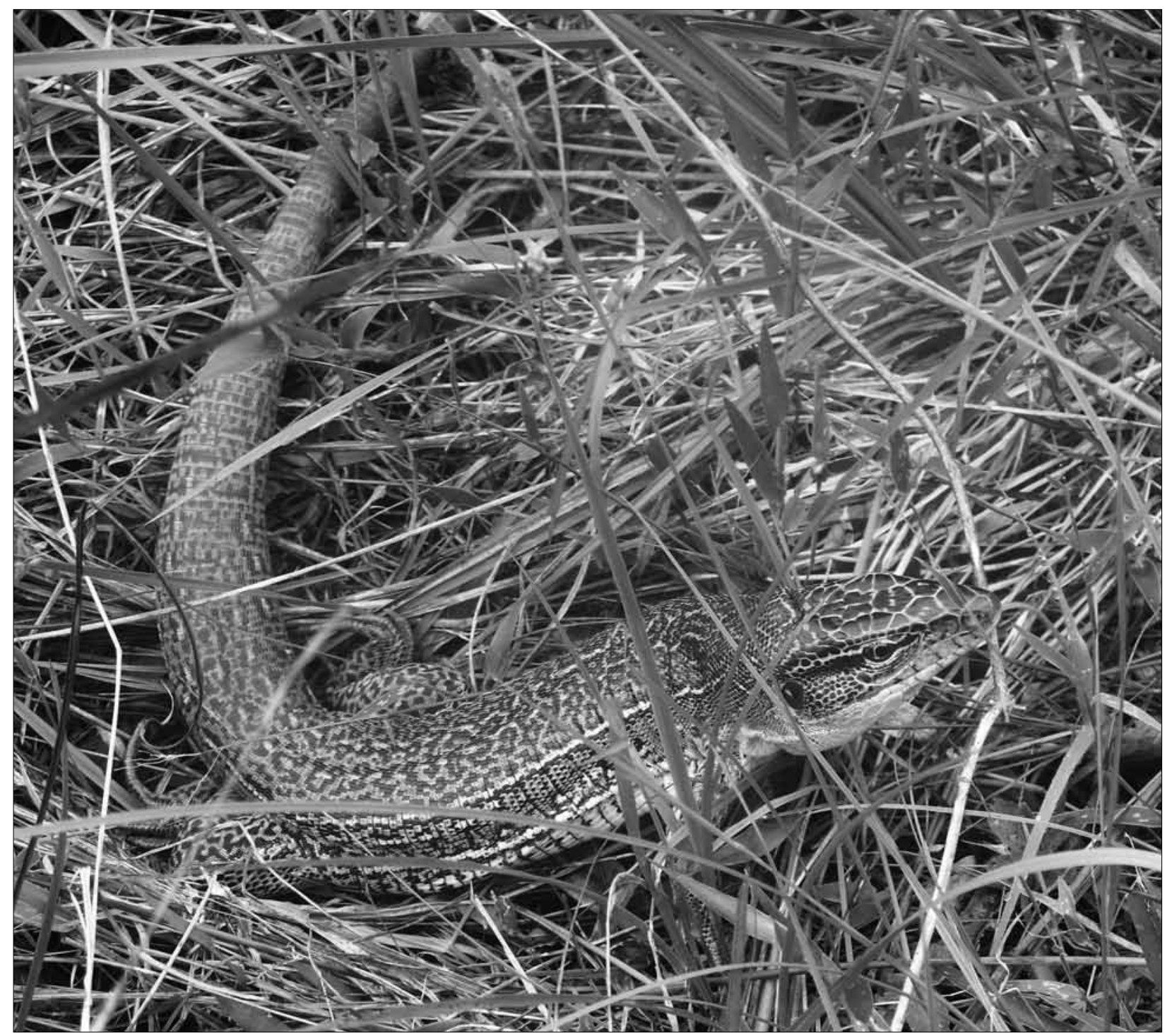

Figura 3. Tupinambis quadrilineatus Manzani \& Abe, 1997 em seu habitat natural. Foto: Leandro Ferreira (MPEG).

estado do Pará, entre os municípios de Redenção e Santa Maria das Barreiras, reforça a ideia de que o conhecimento sobre a distribuição de espécies na Amazônia é ainda limitado.

Este estudo recomenda que parte dos fragmentos de cerrado situados nesta região sejam priorizados para a criação de novas unidades de conservação no âmbito do Projeto Zoneamento Ecológico-Econômico da Zona Leste e Calha Norte do estado do Pará.

\section{AGRADECIMENTOS}

A Luiz Carlos B. Lobato e Lucivaldo S. da Silva, funcionários do Museu Paraense Emílio Goeldi, pelo auxilio na coleta de dados; à Secretaria de Estado de Projetos Estratégicos (SEPE) do estado do Pará, em nome da Sra. Carmen Roseli Caldas Menezes, coordenadora de Ordenamento Territorial do programa Pará Rural, responsável pelo Convênio de Cooperação Técnica n. 003/2008, do Núcleo de Gerenciamento do Programa Pará Rural e do Museu Paraense Emílio Goeldi. 


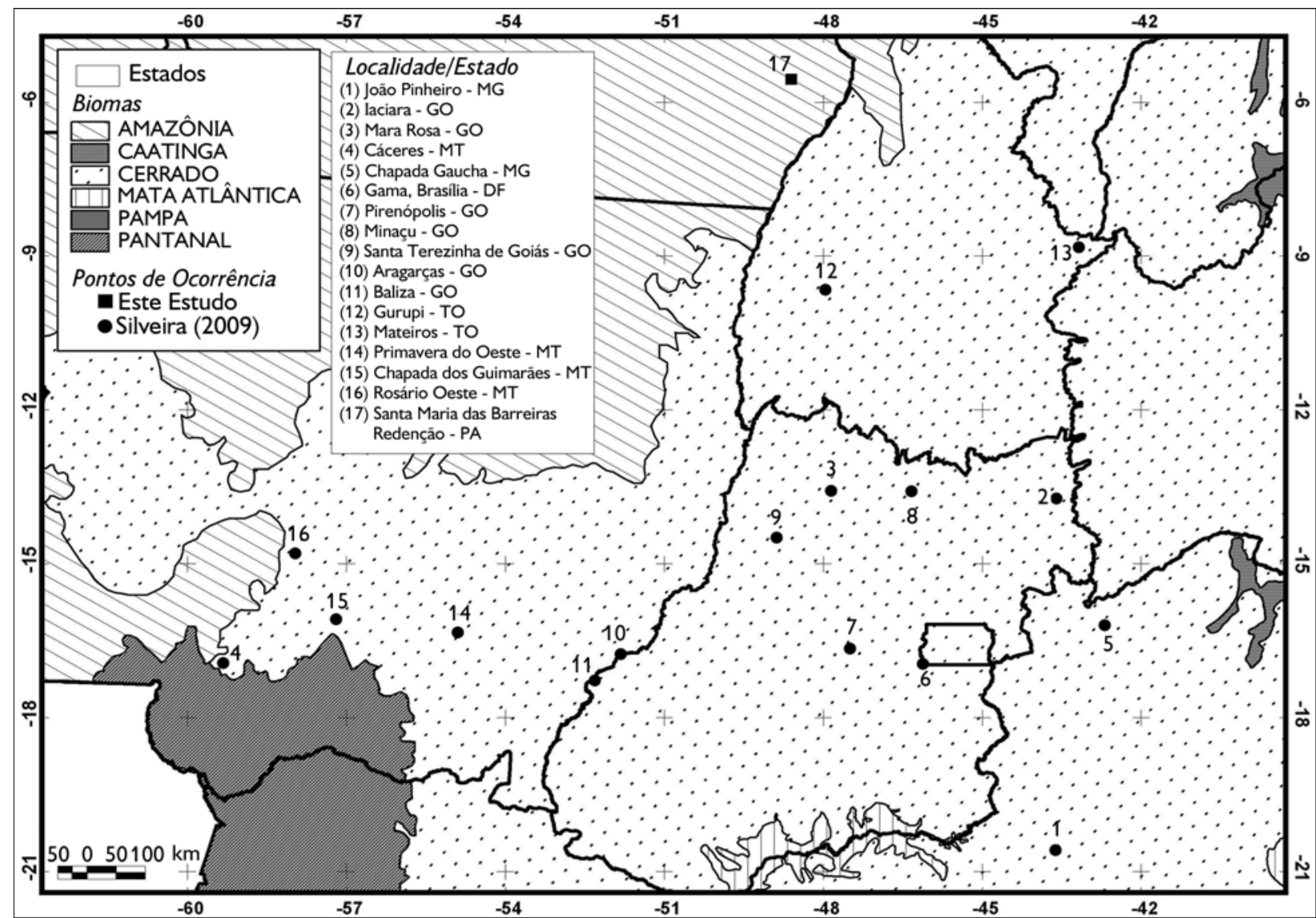

Figura 4. Mapa mostrando a distribuição dos pontos de ocorrência atual e o registrado neste estudo para a espécie Tupinambis quadrilineatus Manzani \& Abe, 1997 (Modificado de Silveira, 2009)

\section{REFERÊNCIAS}

AVILA-PIRES, T. C. S, 1995. Lizards of Amazonian Brasil. Zoologische Verhandelingen 299: 1-706.

COLLI, G. R., A. K. PÉRES JR. \& H. J. CUNHA, 1998. A new species of Tupinambis (Squamata: Teiidae) from Central Brazil, with an analysis of morphological and genetic variation in the genus. Herpetologica 54(4): 477-492.

LEWINSOHN, T. \& P. I. PRADO, 2002. Biodiversidade brasileira: síntese do estado atual do conhecimento: 1-176. Editora Contexto, São Paulo.

MANZANI, P. R. \& A. S. ABE, 1997. A new species of Tupinambis Daudin, 1802 (Squamata: Teiidae) from Central Brazil. Boletim do Museu Nacional, nova série, Zoologia 382: 1-10.

MESQUITA, D. O., G. R. COLLI, F. G. R. FRANÇA \& L. J. VITT, 2006. Ecology of a cerrado lizard assemblage in the Jalapão region of Brazil. Copeia 2006(3): 460-471.
SEMA, 2009. Secretaria do Estado de Meio Ambiente. Macrozoneamento Ecológico-Econômico do Estado do Pará. Disponível em: <http://www.sema.pa.gov.br/macrozoneamento. htm >. Acesso em: 10 junho 2009.

SILVA, M. N. F., A. B. RYLANDS \& J. L. PATTON, 2001. Biogeografia e conservação da mastofauna na floresta Amazônica Brasileira: 110-131. In: J. P. R. CAPOBIANCO, A. VERÍSSIMO, A. MOREIRA, D. SAWYER, I. SANTOS \& L. P. PINTO (Eds.): Biodiversidade na Amazônia Brasileira: avaliação e ações prioritárias para a conservação, uso sustentável e repartição de benefícios. Instituto Socioambiental, São Paulo.

SILVEIRA, A. L., 2009. Notes on geographic distribution: Reptilia, Squamata, Teiidae, Tupinambis quadrillineatus: Distribution extension and geographic distribution map. Check List 5(3): 442-445.

VELOSO, H. P., A. L. R. RANGEL FILHO \& J. C. A. ALVES, 1991. Classificação da vegetação brasileira, adaptada a um sistema universal: 1-123. IBGE, Rio de Janeiro.

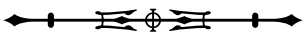


VITT, L. J., J. P. CALDWELL, G. R. COLLI, A. A. GARDA, D. O. MESQUITA, F. G. R. FRANÇA, D. B. SHEPARD, G. C. COSTA, M. M. VASCONCELLOS \& V. N. SILVA, 2005. Uma atualização do guia fotográfico dos répteis e anfíbios da região do Jalapão no cerrado brasileiro. Special Publications in Herpetology, Sam Noble Oklahoma Museum of Natural History 2: 1-24.
ZEE, 2004. Macrozoneamento Ecológico Econômico do estado do Pará - Proposta para a discussão: 1-132. Secretaria Executiva de Ciência, Tecnologia e Meio Ambiente (SECTAM), Pará. Relatório Técnico.

Recebido: 09/06/2009

Aprovado: 14/12/2009

Responsabilidade editorial: Marinus Hoogmoed 
